\title{
Intensity distribution in rotational line spectra. I. Experimental results for Doppler-free $S_{1} \leftarrow S_{0}$ transitions in benzene
}

\author{
H. Sieber, E. Riedle, and H. J. Neusser \\ Institut für Physikalische und Theoretische Chemie, Technische Universität München, Lichtenbergstr. 4, \\ D-8046 Garching, West Germany
}

(Received 23 December 1987; accepted 29 June 1988)

\begin{abstract}
Completely resolved Doppler-free rotational line spectra of six vibronic two-photon bands in benzene $\mathrm{C}_{6} \mathrm{H}_{6}$ and $\mathrm{C}_{6} \mathrm{D}_{6}$ are presented. The excited final states possess different excess energies in $S_{1}$ ( 1567 to $2727 \mathrm{~cm}^{-1}$ ) and are embedded in dense manifolds of background states with differing densities of states $\left(1<\rho<601 / \mathrm{cm}^{-1}\right)$. The bands are analyzed by a statistical procedure. The intensity distribution of several hundreds of lines of each band is investigated. It is found that all weakly perturbed bands display a similar, peaked intensity distribution while in strongly perturbed bands the number of lines decreases monotonically with increasing intensity. The origin of this difference is discussed in terms of coupling to the many background states.
\end{abstract}

\section{INTRODUCTION}

For the understanding of the fundamental processes leading to an intramolecular energy redistribution in large molecules' it is necessary to excite single defined quantum states of the molecule under investigation. After preparation of the molecular system in a defined rovibronic quantum state, selective coupling processes and the redistribution of energy can be observed.

Recently, it has been shown that the rotational band contour of large polyatomic molecules can be completely resolved by Doppler-free techniques. ${ }^{2,3}$ Elimination of the Doppler broadening by Doppler-free two-photon spectroscopy of gaseous benzene at room temperature leads to rotational line spectra containing several thousands of lines. ${ }^{4}$

The analysis of the line spectrum of the $14_{0}^{1}$ band in $\mathrm{C}_{6} \mathrm{H}_{6}$ has shown that perturbations occur at several positions in the line spectrum. ${ }^{5}$ Rotational perturbations are due to a coupling of the "light" $14^{1}$ zero order state to a "dark" vibrational background state in $S_{1}$. This background state has recently been identified by frequency-resolved emission spectroscopy. ${ }^{6}$ In benzene Coriolis coupling rather than anharmonic coupling appears to be responsible for the perturbations. The coupling of the two states results in a splitting of several $100 \mathrm{MHz}$ of the two quasieigenstates. ${ }^{5}$ For the extremely small coherence width of our high resolution $\mathrm{cw}$ laser measurements both quasieigenstates are well resolved. In the $14_{0}^{1}$ band at low excess energy, perturbations are scarce, so that $90 \%$ of all the rotational lines in the spectrum can be assigned by a symmetric rotor approximation including quartic centrifugal distortion. Perturbed lines are then readily identified by deviations from the theoretically predicted positions.

In a polyatomic molecule the size of benzene, the density of vibrational background states increases rapidly with vibrational excess energy. For increasing excess energy, perturbations are expected to become more frequent and ultimately many states might be located within the range of the coupling matrix element. This would lead to a strongly perturbed spectrum whose analysis and assignment is extremely difficult or even impossible. Even though conventional spectroscopy might no longer be possible, this energy range is of particular interest in reaction dynamics' and it might display the transition from a regular to an irregular spectrum. ${ }^{8}$ The understanding of this transition is a basic question in the physical description of large systems with many degrees of freedom. Studies can, in principal, both be performed for highly excited vibrational levels of the electronic ground state or an excited electronic state.

In small molecules the resolution of individual quantum states is possible by Doppler-limited techniques. However, direct access to vibrational states in regimes with the necessary density of states is hardly possible for the electronic ground state due to the low probability of such high overtone transitions and for the electronically excited states due to the small Franck-Condon factor. The necessary high excess energy (in $S_{0}$ ) could only be reached so far by special techniques like stimulated emission pumping (SEP). Experiments have been performed on formaldehyde in the excess energy range from 7400 to $8600 \mathrm{~cm}^{-1}$ and a density of states of $0.151 / \mathrm{cm}^{-19}$ and for acetylene at $\approx 26500 \mathrm{~cm}^{-110}$ and $27900 \mathrm{~cm}^{-111}$ and a density of $\approx 1001 / \mathrm{cm}^{-1}$. 11

Irregular behavior might also be expected for interstate coupling to other electronic states. Experimental results are now available for $\mathrm{NO}_{2},{ }^{12,13}$ glyoxal, ${ }^{14}$ formaldehyde, ${ }^{15}$ and pyrazine. ${ }^{16}$ Indications of irregular behavior were found in these investigations by statistical analysis like nearest-neighbor evaluation, ${ }^{11,12}$ Fourier-transform techniques, ${ }^{10,17}$ and distribution of coupling elements ${ }^{14}$ and line intensities. ${ }^{7,13,16}$ This information pertains so far only to small polyatomic molecules. It is, however, not clear whether the results can also be applied to larger polyatomic molecules. It is therefore desirable to study this situation for larger polyatomic systems, where the critical energy range is still within the Franck-Condon range of the electronic spectrum.

In this work we would like to present rotational line spectra of transitions in benzene, $\mathrm{C}_{6} \mathrm{H}_{6}$ and $\mathrm{C}_{6} \mathrm{D}_{6}$, leading to an excess energy range between 2000 and $3000 \mathrm{~cm}^{-1}$ in $S_{1}$, where the density of states is so high that rotational line spectra are expected to be strongly perturbed. All bands pre- 
sented in this work are well below the "channel three" region, ${ }^{18}$ where a coupling to strongly broadened background states and strong line broadening occurs. ${ }^{3,19,20}$ Since for several of the bands an assignment of rotational lines is no longer possible, an alternative method will be presented which is supposed to characterize the degree of perturbance. This includes the statistical analysis of line intensities. The theoretical background is presented in part II of this work. ${ }^{21}$

\section{EXPERIMENTAL PROCEDURE}

\section{A. Scan of the spectra}

The experimental setup for Doppler-free two-photon spectroscopy of polyatomic molecules has been described in detail in previous work. ${ }^{3}$ Briefly, the ultrahigh resolution is provided by the narrow bandwidth ( $\approx 1 \mathrm{MHz}$ ) light of a $\mathrm{Kr}^{+}$laser-pumped ring dye laser (Coherent CR 699). The light is coupled into an external cavity which contains the fluorescence cell with benzene vapor under a pressure of about 1 Torr. Two-photon excitation to the $S_{1}$ state is monitored by the UV emission from the excited state. In this way a two-photon excitation spectrum is obtained. Doppler-free two-photon absorption is feasible within the standing wave field in the external cavity by simultaneous absorption of two photons with opposite direction of propagation. ${ }^{22}$ Differing from the original setup ${ }^{3}$ the length of the cavity is now locked to the frequency of the laser light by the method of Hänsch and Couillaud ${ }^{23}$ and synchronously varied with the dye laser frequency. A finesse of 30 is achieved with an input mirror of $90 \%$ reflectivity. This finesse leads to a light power of $3 \mathrm{~W}$ within the cavity. As the two-photon absorption efficiency depends quadratically on the laser intensity the increase of the light power within the cavity leads to a twophoton signal nearly three orders of magnitude higher than without external resonator. The resulting good signal to noise ratio is of particular importance for the line intensity statistics performed in this work. The typical dynamic range in our measurements is three orders of magnitude. A high dynamic range is a crucial precondition for line intensity investigations and for the analysis of spectral perturbations as transitions to quasieigenstates with small contribution of the light zero-order state will only result in small intensity lines.

The width of the rotational lines of about $15 \mathrm{MHz}$ is mainly given by the pressure broadening of $15 \mathrm{MHz} /$ Torr. $^{3}$ The average distance of rotational lines in the $Q$ branch of the observed totally symmetric two-photon bands is 250 $\mathrm{MHz}$ so that the resolution of nearly every line is guaranteed for the observed linewidth.

Relative frequency calibration of the Doppler-free spectra is obtained from the simultaneous recording of the transmission of a highly stable $150 \mathrm{MHz}$ Fabry-Perot Interferometer. For absolute calibration of frequency a commercial wavemeter (Burleigh WA 20) with an accuracy of \pm 0.02 $\mathrm{cm}^{-1}$ in the visible is used or a comparison with the simultaneously scanned iodine absorption spectrum ${ }^{24,25}$ is performed.

Experiments were performed with linearly polarized light. For this reason the absorption of two photons with equal direction of propagation is half as probable as that of two photons with opposite direction and produces a Doppler-broadened background with a frequency width of $1.7 \mathrm{GHz}$. Due to its broadness the intensity of the background is only less than $1 \%$ of the height of the high intensity sharp Doppler-free lines. However, for bunches of lines the Doppler-broadened background might become larger than small lines which may be located within this bunch. The small lines are then small peaks on top of the Doppler-broadened background (see, e.g., the bunch of lines at $-145 \mathrm{GHz}$ in Fig. 2). This causes some difficulties for the computerassisted determination of the small line intensities since there exists no flat base line. Particularly, the small lines are important for the physical interpretation of the line intensity statistics. ${ }^{7}$

\section{B. Data analysis}

Even with the external cavity the UV emission of the excited benzene molecules is not sufficient to produce an analog signal. Therefore, single photon counting is employed and photons are counted for intervals of $20 \mathrm{~ms}$, corresponding to a change in two-photon energy of typically 1.5 $\mathrm{MHz}$ during the laser frequency scan. The raw data $x_{i}$ are stored in a minicomputer for consequent evaluation of the spectra. Small statistical fluctuations of the count rate would complicate the determination of the maxima in the observed spectrum, i.e., the position and the height of the lines. Therefore, the raw data are smoothed by convolution with a normalized Gaussian function $\boldsymbol{G}(k)$ of five points FWHM

$$
y_{i}=\sum_{j} x_{j} \cdot G(i-j) .
$$

This convolution does not seriously increase the width of the lines but is sufficient to allow reliable determination of lines by computer search for points $y_{i}$ that satisfy the condition

$$
\begin{aligned}
y_{i-4} & <y_{i-3}<y_{i-2}<y_{i-1}<y_{i} \\
>y_{i+1} & >y_{i+2}>y_{i+3}>y_{i+4} .
\end{aligned}
$$

The exact height of the maximum is then determined by fitting a parabola to the five values $y_{j}$ centered around the position of the maximum $y_{i}$ and the maximal value of this parabola is consequently used for the height of the maximum. To find the intensity of the line, the contribution of the varying Doppler-broadened background to the signal has to be taken into account. For this purpose the two minimal values of $y_{j}$ in the range of 2.5 FWHM to each side of the maximum are searched and their average value is subtracted from the height of the maximum. The intensity of each line is stored together with its frequency position.

For the statistical analysis of the line intensities, the rotational lines are sorted according to their height and the number of lines per intensity interval is counted. The size of each intensity interval is chosen according to the following considerations in a way that allows us to compare the statistics of all presented spectra. The couplings responsible for the deviation of the rotational structure from a regular sym- 
metric top model are known to produce only relatively small splittings of the lines. ${ }^{5}$ The sum of the intensities of all transitions to quasieigenstates with contribution from one light zero-order state is equal to the expected intensity of the transition to the light state and there is no additional contribution from the coupled dark states. Since frequency intervals much larger than the splittings are analyzed, the sum of all intensities should therefore not be changed by the perturbations. However, differing experimental conditions and vibronic transition strengths for the various bands under investigation do cause changes of the total intensity. For this reason we decided to normalize the intensity intervals according to the sum of the intensities of all lines in the frequency interval from 0 to $-120 \mathrm{GHz}$ from the rotationless origin. For comparison of spectra of $C_{6} H_{6}$ and $C_{6} D_{6}$, the sum of the intensities is in addition weighted by the ratio of $\Delta B$ for the two isotopes, as $\Delta B$ is known to determine the density of lines in the analyzed $Q$ branch spectra. The average intensity of the rotational lines in a regular spectrum was chosen as unit intensity [see Figs. 4(a) and 4(b)]. The size of the intensity intervals is 0.05 of this intensity.

\section{RESULTS AND DISCUSSION}

\section{A. Purity of the spectra}

Before starting a line intensity analysis it is important to select suitable parts of the spectrum. It has to be checked whether contributions from accidentally overlapping bands can be excluded. Contributions from isotopic impurities will be discussed in common below, while overlapping bands originating from the molecule itself will be discussed for each band separately. If these checks have been performed, the vibrational quantum of the light zero order state can be unambiguously defined and other vibronic states can only influence the intensity distribution via an intramolecular coupling process.

\section{Selection of the spectral region}

In a monochromatic light field the two-photon transition is due to two contributions with different polarization behavior. ${ }^{26,27}$ These are the isotropic and the symmetric anisotropic parts of the two-photon absorption tensor. The isotropic part of the two-photon tensor of benzene for parallel linear polarization $(\downarrow \uparrow)$ of the two absorbed photons is larger by more than one order of magnitude than its anisotropic part. ${ }^{26}$ The strong isotropic contribution does only exist for the $Q$ branch of totally symmetric transitions, the only type of bands investigated in this work. For this reason, the $Q$ branch is by far the strongest part of the observed bands.

The lines of the $Q$ branch ( $\Delta J=0$ ) of a totally symmetric band (with $\Delta K=0$ ) are found to the blue side of the rotationless origin. Their distance from the rotationless origin is proportional to the ground state rotational energy and no Fortrat parabola turning point occurs, which would lead to a congestion of the rotational lines. In particular, the $Q$ branch lines found in the range from 0 to $-120 \mathrm{GHz}$ from the rotationless origin correspond to relatively small $J$ and $K$ rotational quantum numbers. Due to the relative strength of the $Q$ branch (see above) the intensity of the lines of the $O, P$, $R$, and $S$ branches in this range is only less than $1.5 \%$ of the strongest $Q$ branch line, i.e., all strong lines observed in the range from 0 to $-120 \mathrm{GHz}$ orginate from one rotational branch, the $Q$ branch.

Since the range from 0 to $-120 \mathrm{GHz}$ is expected to contain only $Q$ branch lines with low rotational quantum numbers, it is also the one part of the spectrum, that could be assigned most easily, if such an assignment is possible at all. In addition, the rotational structure in this range does not depend very strongly on small changes in the rotational constants, as they are typical for various vibronic bands, and thus the comparison of different bands is meaningful.

In summary, in the range of 0 to $-120 \mathrm{GHz}$ from the rotationless origin:

(i) All strong rotational lines belong to the $Q$ branch and have low rotational quantum numbers $(J$, $K \leqslant 30$ ).

(ii) There is no congestion due to a turning point of a Fortrat parabola.

(iii) An assignment, if possible at all for a band, should be easiest in this range.

(iv) The rotational structure of different vibronic bands of the same symmetry should be very similar.

For these reasons the blue edge of the $Q$ branch ( 0 to $-120 \mathrm{GHz}$ from the rotationless origin) was chosen for the statistical analysis.

\section{Isotopic impurities}

Natural benzene contains about $6 \%$ of "heavy" mono${ }^{13} \mathrm{C}$-benzene due to the natural isotopic abundance of ${ }^{13} \mathrm{C}$. The vibronic two-photon bands of heavy benzene are shifted by several wave numbers from the vibronic bands of light benzene. For most of the bands of $C_{6} H_{6}$ and $C_{6} D_{6}$ investigated in this work, the isotopic shifts are known from Doppler-limited measurements in a seeded supersonic beam at low temperature. ${ }^{28}$ It was found that isotopic substitution leads to a red shift of the vibronic bands of several wave numbers. Due to the red shift there is no interference of the isotopic spectra in the selected parts $(0$ to $-120 \mathrm{GHz}$ to the red of the rotationless origin) of the two-photon spectra. Lines of heavy benzene isotopes which may contribute to the spectrum can only be due to the anisotropic contributions of the $R$ branch and $S$ branch and are therefore smaller than $0.1 \%$ of the strongest lines of light benzene. They are not considered in the intensity statistics.

\section{B. Density of background states}

In a molecule the size of benzene with 30 vibrational degrees of freedom, there are many combination states whose density increases rapidly with excess energy in $S_{1}$. In Fig. 1 the calculated total density of background states in harmonic approximation is plotted as a function of excess energy for $\mathrm{C}_{6} \mathrm{H}_{6}$ and $\mathrm{C}_{6} \mathrm{D}_{6}$ on a half-logarithmic scale. The harmonic frequencies of $\mathrm{C}_{6} \mathrm{H}_{6}$ were taken from the compilation of Stephenson et al..$^{29}$ and supplemented by calculated frequencies, ${ }^{30}$ where no experimental values were available. For $C_{6} D_{6}$, only calculated frequencies ${ }^{30}$ were used. The irre- 


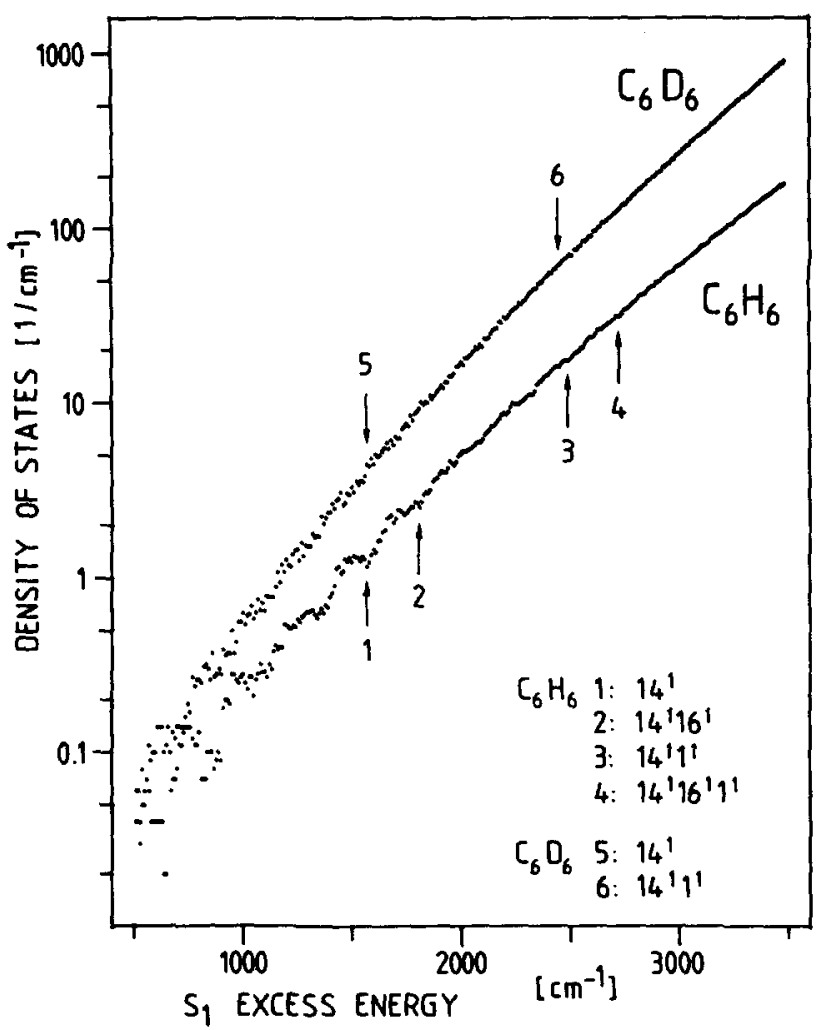

FIG. 1. Total density of vibrational states in benzene $C_{6} H_{6}$ and $C_{6} D_{6}$ as a function of excess energy in $S_{1}$. The excess energy of each band whose Doppler-free spectrum is presented and investigated in this work is marked by an arrow. ducible representation of each combination state was determined in an exact way ${ }^{31}$ to evaluate the degree of degeneracy. Due to the smaller vibrational frequencies in $C_{6} D_{6}$ the density of states increases even faster for $C_{6} D_{6}$ than for $\mathrm{C}_{6} \mathrm{H}_{6}$. At $2500 \mathrm{~cm}^{-1}$ excess energy it is already $701 / \mathrm{cm}^{-1}$ and a factor of 4 higher than the corresponding density of states in $\mathrm{C}_{6} \mathrm{H}_{6}$. All bands investigated in this work are marked by an arrow in Fig. 1.

\section{Line intensity distribution of weakly perturbed bands}

\section{1. $14_{o}^{t}$ band in $\mathrm{C}_{6} \mathrm{H}_{6}$}

The blue edge of the $Q$ branch of the $14_{1}^{0}$ band with its rotationless origin $v_{00}$ at $39656.90(5) \mathrm{cm}^{-1}$ in $\mathrm{C}_{6} \mathrm{H}_{6}$ is shown in Fig. 2. This band was analyzed in great detail. ${ }^{32}$ $90 \%$ of all lines are unpertubed and are exactly at the position expected for a semirigid symmetric top molecule. The rotational and quartic centrifugal distortion constants found from a fit to more than 3000 lines from the $Q, R$, and $S$ branch are listed in Table $I$. The accuracy of the constants is determined by the statistical error in the fit and the accuracy of the frequency calibration.

In Fig. 3 part of the experimental spectrum (lower trace) shown in Fig. 2 is compared to a theroretical one (upper trace), that was calculated with the constants of Table I and a Lorentzian linewidth of $15 \mathrm{MHz}$. The calculated spectrum represents an absorption spectrum, but it reflects the rotational structure of the two-photon excitation spectrum as long as no coupling to dark background states in $S_{1}$
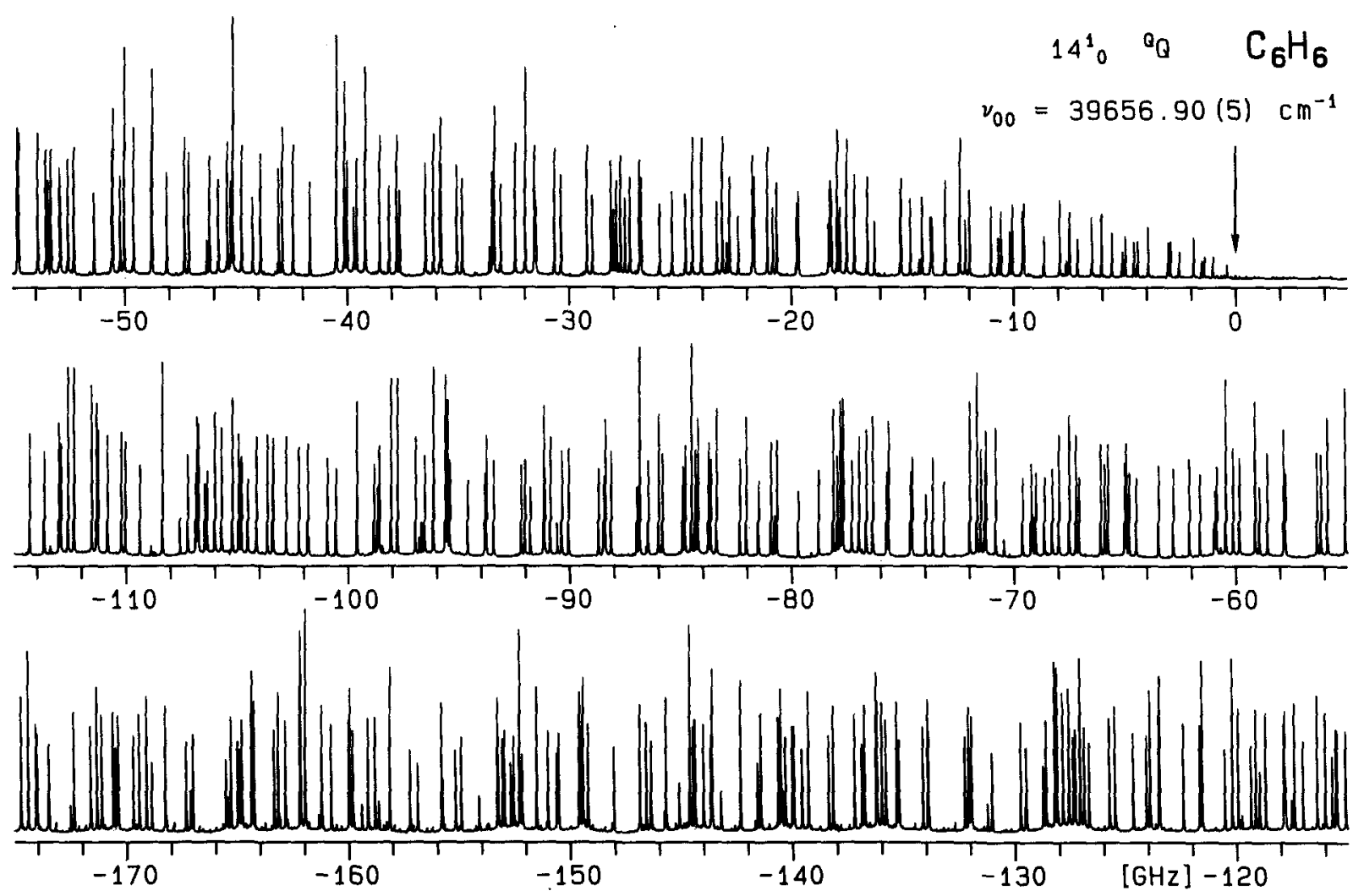

FIG. 2. Part $\left(6 \mathrm{~cm}^{-1}\right)$ of the $Q$ branch in the Doppler-free two-photon spectrum of the $14_{0}^{1}$ band in $\mathrm{C}_{6} \mathrm{H}_{6}$. The rotationless origin $v_{00}$ is marked by an arrow. 
TABLE I. Rotational constants and quartic centrifugal distortion constants for $\mathrm{C}_{6} \mathrm{H}_{6}$ (Ref. 32) and $\mathrm{C}_{6} \mathrm{D}_{6}$ (Ref. 35) obtained from the analysis of the Doppler-free $Q$ branch spectrum of the $14_{0}^{1}$ band $\left(\mathrm{cm}^{-1}\right)$. From the $Q$ branch only the changes in the constants due to the electronic excitation can be determined. The accuracy is given by the statistical error in the fit and the accuracy of the calibration of the spectra.

\begin{tabular}{llc}
\hline \hline & $C_{6} \mathrm{H}_{6}$ & $\mathrm{C}_{6} \mathrm{D}_{6}$ \\
\hline$\Delta B=B_{14}^{\prime}-B_{0}^{\prime \prime}$ & $-0.0084782(2)$ & $-0.006490(6)$ \\
$\Delta C=C_{14}^{\prime}-C_{0}^{\prime \prime}$ & $-0.00416987(9)$ & $-0.003235(5)$ \\
$\Delta D_{J}=D_{J, 14}^{\prime}-D_{J, 0}^{\prime \prime}$ & $+4.64(4) * 10^{-9}$ & \\
$\Delta D_{J K}=D_{j K, 14}^{\prime \prime}-D_{J K, 0}^{\prime \prime}$ & $-8.85(7) * 10^{-9}$ & \\
$\Delta D_{K}=D_{K, 14}^{\prime}-D_{K, 0}^{\prime \prime}$ & $+4.12(4) * 10^{-9}$ & \\
\hline
\end{tabular}

occurs and the fluorescence quantum yield is independent of the molecular rotation. In our recent single quantum state decay time measurements we have shown that decay time and hence the fluorescence quantum yield does indeed not change with the rotational quantum numbers $J, K$ for unperturbed states. ${ }^{33}$ This has been explained by a $S_{1}-T_{1}$ coupling in the statistical limit. Very good agreement between the calculated and the experimental spectrum is found for the $Q$ branch lines.

At higher rotational energies isolated perturbations are found in the experimental spectrum. $10 \%$ of the lines are perturbed due to a coupling to dark background states. The $14^{1}$ state is located at $1571 \mathrm{~cm}^{-1}$ excess energy in $S_{1}$. The total density of background vibrational states is
$11 / \mathrm{cm}^{-1}$ and rather low. For this reason the coupling is very selective and the coupled background states were identified by observation of dispersed emission spectra from single quantum states. $^{6}$

In Fig. 3 (lower trace), also part of the $14_{0}^{1}$ band on the blue side of the rotationless origin (marked by an arrow) is shown and it can be seen that neither contributions from other vibronic bands nor from the anisotropic $R$ branch transition of the $14{ }_{0}^{1}$ band yield lines higher than $1.5 \%$ of the strongest $Q$ branch lines. The $14_{0}^{1}$ band of mono- ${ }^{13} \mathrm{C}$-benzene, present with $6 \%$ abundance in the natural sample, is shifted by $3.3 \mathrm{~cm}^{-1}$ or $100 \mathrm{GHz}$ to the red. ${ }^{28}$ Indeed, around $-120 \mathrm{GHz}$ an increasing number of small lines is seen in Fig. 2 which originates from the isotropic $Q$ branch of this isotopic molecule.

An analysis of the distribution of line intensities was performed for all 277 rotational lines (higher than $1.5 \%$ of the strongest line) located between 0 and $-120 \mathrm{GHz}$ of the experimental spectrum in Fig. 2 and the calculated spectrum (see Fig. 3). The result is shown in Fig. 4(b) for the calculated spectrum and in Fig. 4(d) for the experimental spectrum. The computerized procedure described in Sec. II B was applied to both, the experimental and the theoretical spectrum. The sum of line intensities for the observed 0 to $-120 \mathrm{GHz}$ experimental range is 720224 counts (see Table II). This sum is used for normalization of the distribution as described in Sec. II B to allow the comparison with the line intensity distribution of the calculated spectrum, which represents a totally unperturbed spectrum. We note that both the theo-
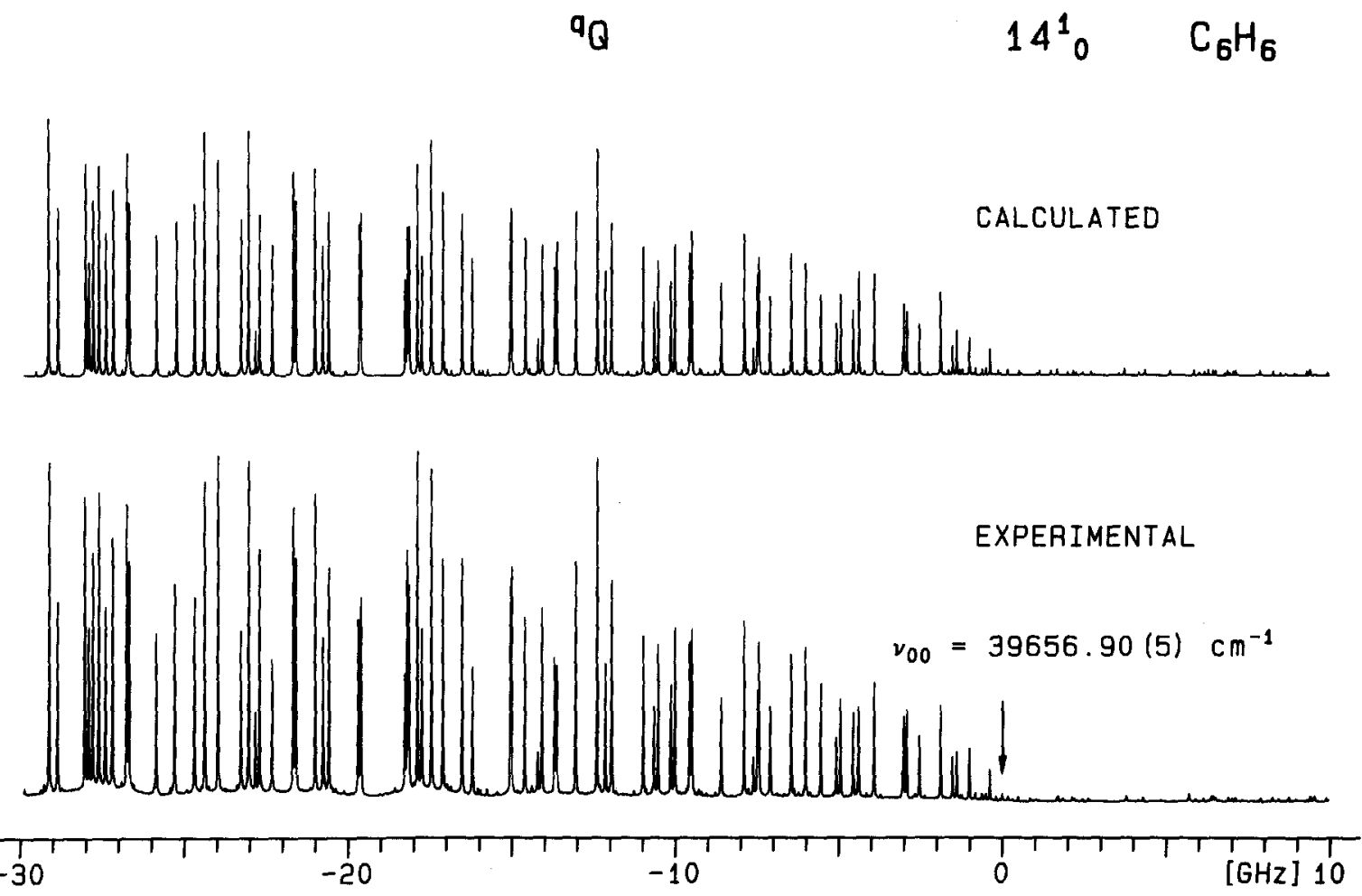

FIG. 3. Lower trace: Magnified portion of the $Q$ branch of the $14_{0}^{1}$ band shown in Fig. 2. Upper trace: same part of the spectrum calculated with the rotational constants and quartic centrifugal distortion constants listed in Table $I$. 


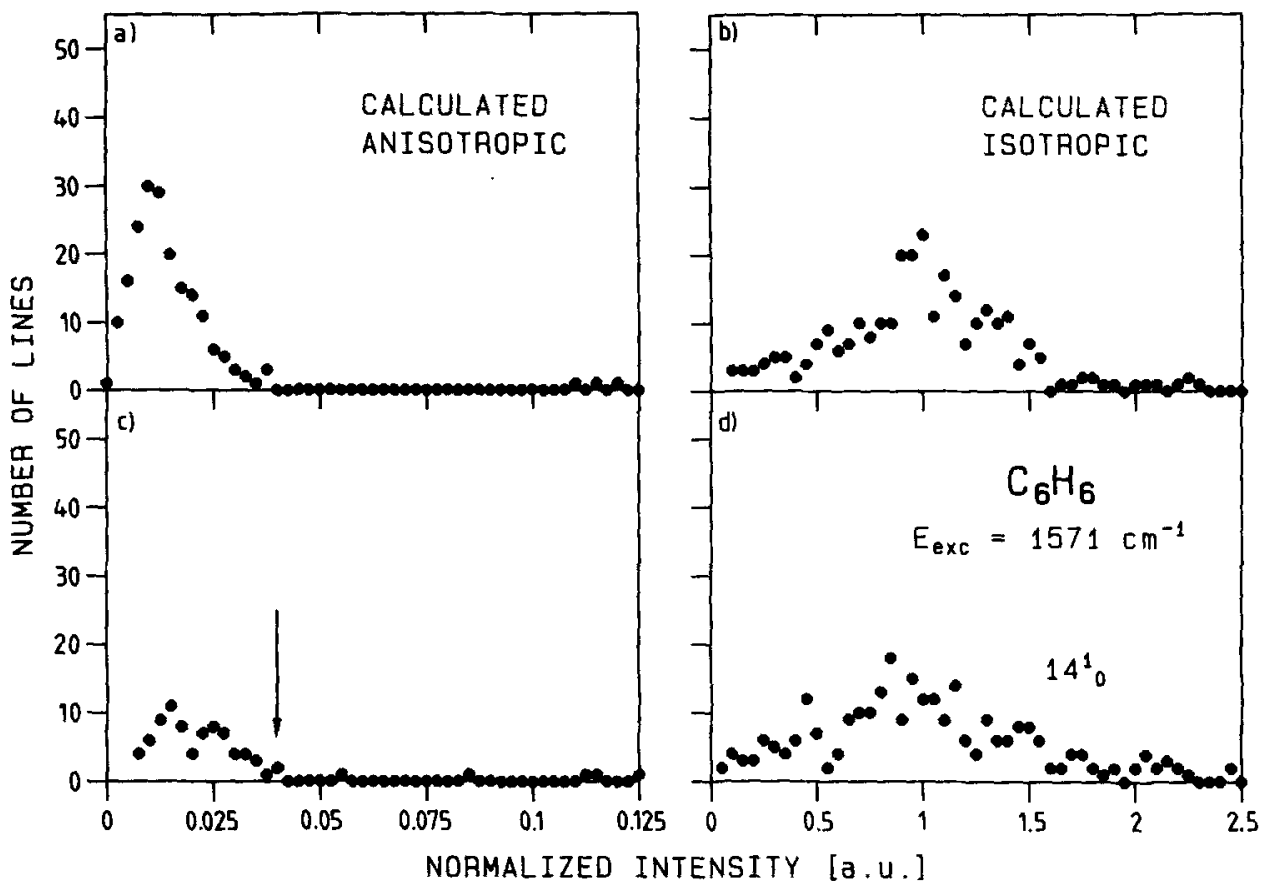

FIG. 4. Intensity distribution of lines between 0 and $-120 \mathrm{GHz}$ : (a),(b): The calculated spectrum shown in part in Fig. 3. The anisotropic and isotropic contributions of the two-photon tensor lead to two sets of lines with strongly differing mean intensities. (c),(d): The experimental spectrum shown in Fig. 2. The intensity scale is chosen so that intensity 1 [a.u.] coincides with the maximum in the distribution of calculated isotropic lines. Note the different intensity scales for anisotropic and isotropic lines. retical and experimental line intensity distributions possess a maximum at 1 a.u., display a decreasing probability for lines with increasing and decreasing intensity, and have nearly symmetric wings. In Figs. 4(a) and 4(c) the intensity range below 0.125 a.u. is shown on a magnified scale. For this distribution even lines lower than $1.5 \%$ were considered. It is seen that a second maximum appears at intensities below 0.04 a.u. This is due to the anisotropic contribution from the $Q, P$, and $R$ branches. This can be seen from a comparison of the experimental and theoretical line intensity distribution in Fig. 4(c). Particularly, the well resolved peak at very small intensities (due to the anisotropic lines) demonstrates the large dynamic range of our experiment. The peak due to isotropic lines in the experimental spectrum is only slightly shifted from 1 a.u. to lower intensities. This reflects the weakly perturbed character of this band and is in line with the somewhat larger low intensity wing of the isotropic peak for the experimental spectrum. Since for the selected part of this band all lines have been identified in the detailed spectroscopic analysis ${ }^{32}$ we can find out which lines contribute to different parts of the intensity distribution. It is seen that perturbed lines are mainly responsible for the low intensity part of the distribution in Fig. 4(d).

From Fig. 4 it is evident that anisotropic parts and isotropic parts of the totally symmetric two-photon bands are well separated in the line intensity distribution. It is easy to suppress the anisotropic contributions when only lines are taken into account in the intensity statistics whose intensity

TABLE II. Summary of all data from the statistical analysis of Doppler-free two-photon bands. For each band the total number of lines found in the interval 0 to $-120 \mathrm{GHz}$, the highest line intensity, and the sum of all intensities is given. In addition, the total density of background states in $S_{1}$ for the excess energy of the final state of each vibronic transition and, if applicable, the assignment of the overlapping band is shown. The type of line intensity distribution found is denoted by $m$ for one maximum, $2 m$ for two maxima, and $p$ for perturbed. "Calculated" refers to a synthetic spectrum obtained from the rotational constants of the $14_{0}^{!}$band of $\mathrm{C}_{6} \mathrm{H}_{6}$ (see also Table I and Fig. 3).

\begin{tabular}{|c|c|c|c|c|c|c|c|}
\hline Band & $\begin{array}{c}\text { Number } \\
\text { of } \\
\text { lines }\end{array}$ & $\begin{array}{l}\text { Excess } \\
\text { energy } \\
\left(\mathrm{cm}^{-1}\right)\end{array}$ & $\begin{array}{c}\text { Density of } \\
\text { states } \\
\left(1 / \mathrm{cm}^{-1}\right)\end{array}$ & $\begin{array}{l}\text { Overlapping } \\
\text { band }\end{array}$ & $\begin{array}{l}\text { Highest } \\
\text { line } \\
\text { intensity }\end{array}$ & $\begin{array}{l}\text { Sum of } \\
\text { line } \\
\text { intensity }\end{array}$ & Distriution \\
\hline $\begin{array}{l}\mathrm{C}_{6} \mathrm{H}_{6}: \\
14_{0}^{1}\end{array}$ & 277 & 1571 & 1 & & 6645 & 720224 & $m$ \\
\hline $14_{0}^{1} 16_{1}^{1}$ & 805 & 1806 & 3 & $14_{0}^{1} 11_{1}^{1}$ & 9773 & 1467830 & $2 m$ \\
\hline $14_{0}^{1} 1_{0}^{1}$ & 531 & 2492 & 18 & $14_{0}^{1} 16_{0}^{1} 17_{0}^{1}$ & 35771 & 4935639 & $2 m$ \\
\hline $14_{0}^{1} 1_{0}^{1} 16_{1}^{1}$ & 827 & 2727 & 32 & & 3761 & 494090 & $p$ \\
\hline calculated & 282 & & & & 163 & 17780 & $m$ \\
\hline $\begin{array}{l}C_{6} D_{6}: \\
14_{0}^{!}\end{array}$ & 471 & 1567 & 4 & & 23664 & 3270483 & $m$ \\
\hline $14_{0}^{\prime} 1_{0}^{\prime}$ & 740 & 2445 & 60 & $14_{0}^{1} 10_{0}^{2}$ & 9234 & 1284645 & $p$ \\
\hline
\end{tabular}


is larger than 0.04 a.u. This lower threshold is used for all line intensity distributions in this work and marked by an arrow in Fig. 4(c).

As noted before, all intensity scales used in the line intensity distribution of this work are normalized to the sum of all line intensities in the frequency range 0 to $-120 \mathrm{GHz}$ and can therefore directly be compared with the distributions shown in Figs. 4(b) and 4(d) which are typical for an unperturbed or weakly perturbed band. Deviations from this type of distribution can then be interpreted as due to perturbations of the band.

\section{2. $14_{a}^{1} 16_{1}^{\prime}$ and $14_{o}^{1} 11_{1}^{y}$ band in $\mathrm{C}_{6} \mathrm{H}_{6}$}

In Fig. 5 the line spectrum of the $14_{0}^{1} 16_{1}^{1}$ sequence band in $\mathrm{C}_{6} \mathrm{H}_{6}$ is shown. The rotationless origin $v_{00}$ is located at $39493.25(5) \mathrm{cm}^{-1}$ two-photon energy. The $14^{1} 16^{1}$ state of this transition is at $1806 \mathrm{~cm}^{-1}$ excess energy with a total density of states of $31 / \mathrm{cm}^{-1}$. Due to the congested sequence structure in this spectral region sequence bands begin to overlap. This is clearly seen in Fig. 5 . The series of rotational lines of constant but smaller intensity clearly seen to the blue of the $14_{0}^{1} 16_{1}^{1}$ sequence band and extending into the band belongs to another vibronic band. This is the $14_{0}^{1} 11_{1}^{1}$ band with the rotationless origin at $7.5 \mathrm{~cm}^{-1}$ to the blue of the $14_{0}^{1} 16_{1}^{1}$ band. ${ }^{28}$ This overlap of bands leads to a strongly increased total number of lines in the observed frequency interval 0 to $-120 \mathrm{GHz}$ of the $14_{0}^{1} 16_{1}^{1}$ band. The $14_{0}^{1} 16_{1}^{1}$ sequence band was recently analyzed by Somers et $a l^{34}$ and it was found to be weakly perturbed. However, l-type doubling occurs which leads to a splitting of lines (see insert in Fig. 5). Both, the overlapping $14_{0}^{1} 11_{1}^{1}$ band and the $l$-type doubling results in a three times larger total number of lines (805) as compared to the unperturbed $14_{0}^{1}$ band (277, see Table II) in the same frequency range. For a weakly perturbed band a line intensity distribution similar to that of the $14_{0}^{1}$ band in $\mathrm{C}_{6} \mathrm{H}_{6}$ [see Fig. 4(d) ] is expected. However, the question arises whether the interfering $14_{0}^{1} 11_{1}^{1}$ band leads to a strong distortion of the intensity distribution and may simulate a higher degree of perturbance.

The distribution of line intensities in the interval 0 to $-120 \mathrm{GHz}$ to the red of the rotationless origin of the $14_{0}^{1} 16_{1}^{1}$ band is plotted in Fig. 6 (upper part). The sum of all line intensities used for the normalization of the intensity scale was corrected for the contribution from the $14_{0}^{1} 11_{1}^{1}$ band under the assumption that the line structure of the $14_{0}^{1} 11_{1}^{1}$ band is identical to both sides of the origin of the $14_{0}^{1} 16_{1}^{1}$ band. Therefore, the resulting intensity distribution can be directly compared with those of Fig. 4. It displays two peaks. The one located at line intensity $0.5 \mathrm{a}$.u. is very similar to the peak of the intensity distribution of the $14_{0}^{1}$ band in Fig. 4(d). The intensity of 0.5 a.u. is half of the intensity expected for a completely unperturbed band. The maximum is readily ascribed to the stronger $14_{0}^{1} 16_{1}^{1}$ band and its shape agrees well with the weakly perturbed character found from the spectral analysis of this band. ${ }^{34}$ The shift of the peak
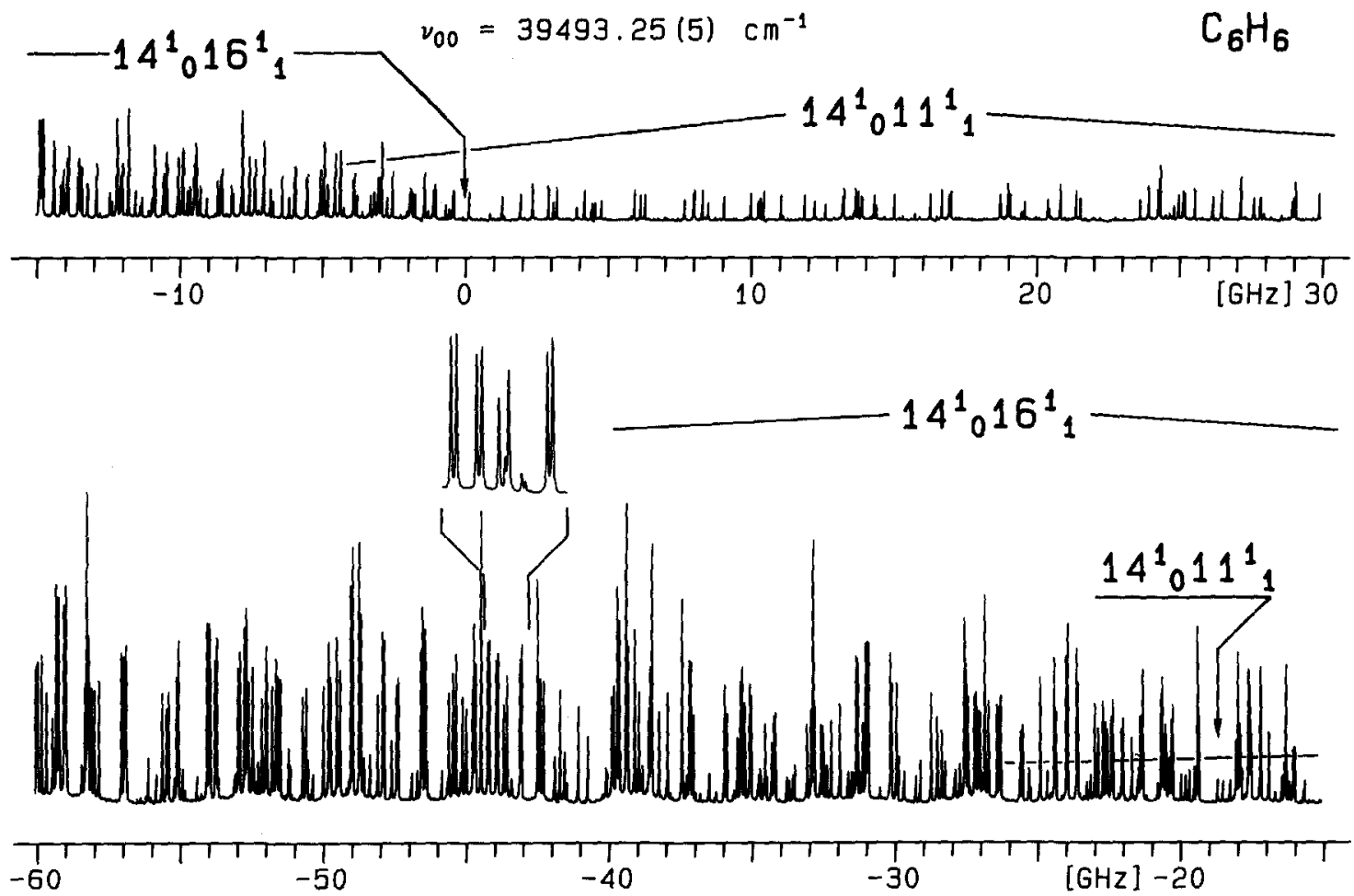

FIG. 5. Part of the Doppler-free two-photon spectrum of the $Q$ branch of the $14_{0}^{1} 16_{1}^{1}$ band in $C_{6} H_{6}$. The rotationless origin $v_{00}$ is marked by an arrow. To its blue side lines from an additional band, $14_{0}^{1} 11_{1}^{1}$, are seen which overlaps with the $Q$ branch of the $14_{0}^{1} 16_{1}^{1}$ band. The magnified portion of the spectrum between 43 and $44.5 \mathrm{GHz}$ demonstrates the $l$-type doubling of all lines in the $14_{0}^{l} 16_{1}^{1}$ band. 
maximum to smaller intensities is due to the $l$-type doubling. This leads to a doubling of each line with two lines of half the intensity (see insert of Fig. 5). At low line intensities, where contributions from perturbed lines are expected a strong peak occurs. This peak is ascribed to the contribution of the overlapping weaker $14_{0}^{1} 11_{1}^{1}$ band. This is proved by the line intensity distribution of lines to the blue of the origin of the $14_{0}^{1} 16_{1}^{1}$ band shown in the lower part of Fig. 6 . These lines can be due only to the overlapping $14_{0}^{1} 11_{1}^{1}$ band. The size of the intensity interval in this distribution was chosen identical to the intervals for the distribution of the 0 to $-120 \mathrm{GHz}$ range and the number of lines per intensity interval was corrected for the fact that only a range of $43.45 \mathrm{GHz}$ containing 106 lines was evaluated. Therefore both the number of lines and the intensity of the distribution of the blue part can be directly compared with the distribution shown in the upper part of Fig. 6. It is seen that the positions and the height of the maxima under consideration are the same. From this we conclude that also the low intensity peak in the upper part of Fig. 6 orginates from the overlapping $14_{0}^{1} 11$ band. It is interesting to see that the contributions of both overlapping bands can be well separated in the line intensity distribution and that the weakly perturbed character of the stronger $14_{0}^{1} 16_{1}^{1}$ band is still apparent.

\section{3. $14_{0}^{1} 1_{0}^{1}$ and $14_{0}^{1} 16_{0}^{1} 17_{0}^{1}$ band in $C_{e} H_{0}$}

The rotationless origin $v_{00}$ of the $14_{0}^{1} 1_{0}^{1}$ band in $\mathrm{C}_{6} \mathrm{H}_{6}$ is located at the two-photon excitation energy of $40578.22(5) \mathrm{cm}^{-1}$. The final $14^{1} 1^{1}$ state has an excess ener-

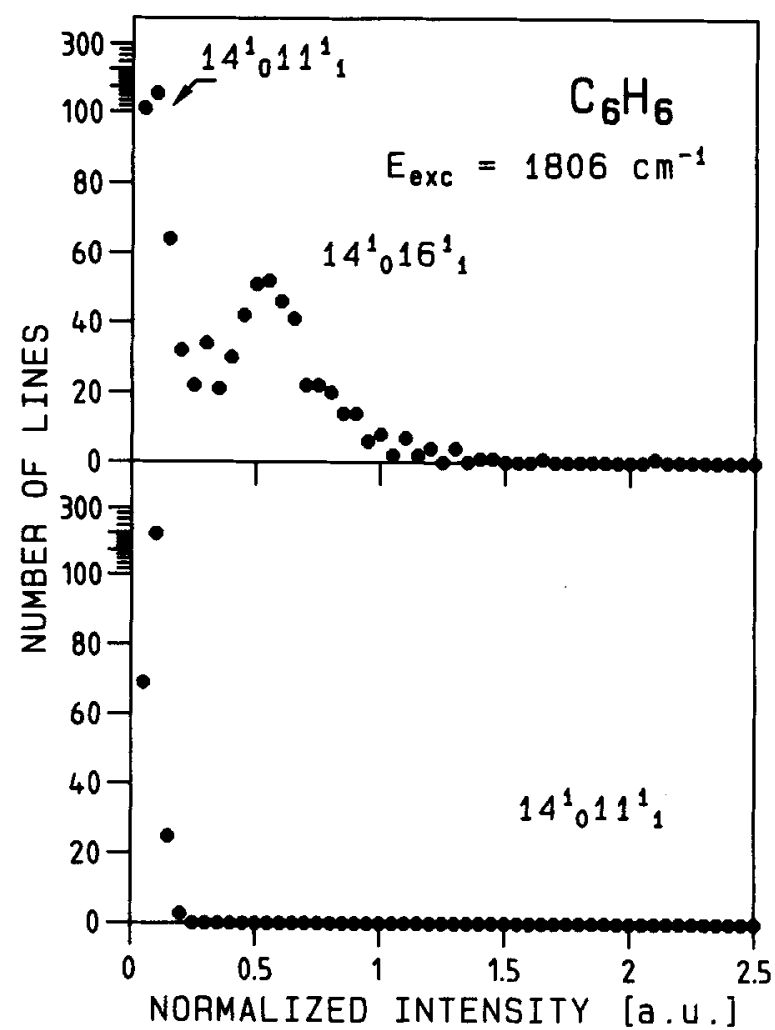

FIG. 6. Intensity distribution of lines between 0 and - $120 \mathrm{GHz}$ : Upper trace: in the $Q$ branch of the $14_{0}^{1} 16_{1}^{1}$ band in $\mathrm{C}_{6} \mathrm{H}_{6}$. The two peaks result from the lines of the two overlapping bands which are indicated in the figure. Lower trace: Of lines located to the blue of the rotationless origin of the $14{ }_{0}^{1} 16_{1}^{1}$ band. Only lines of the $14_{0}^{1} 11_{1}^{1}$ band contribute to this distribution. Note the change in the scale of the vertical axis. For explanation of the intensity scale (horizontal axis) see the text.

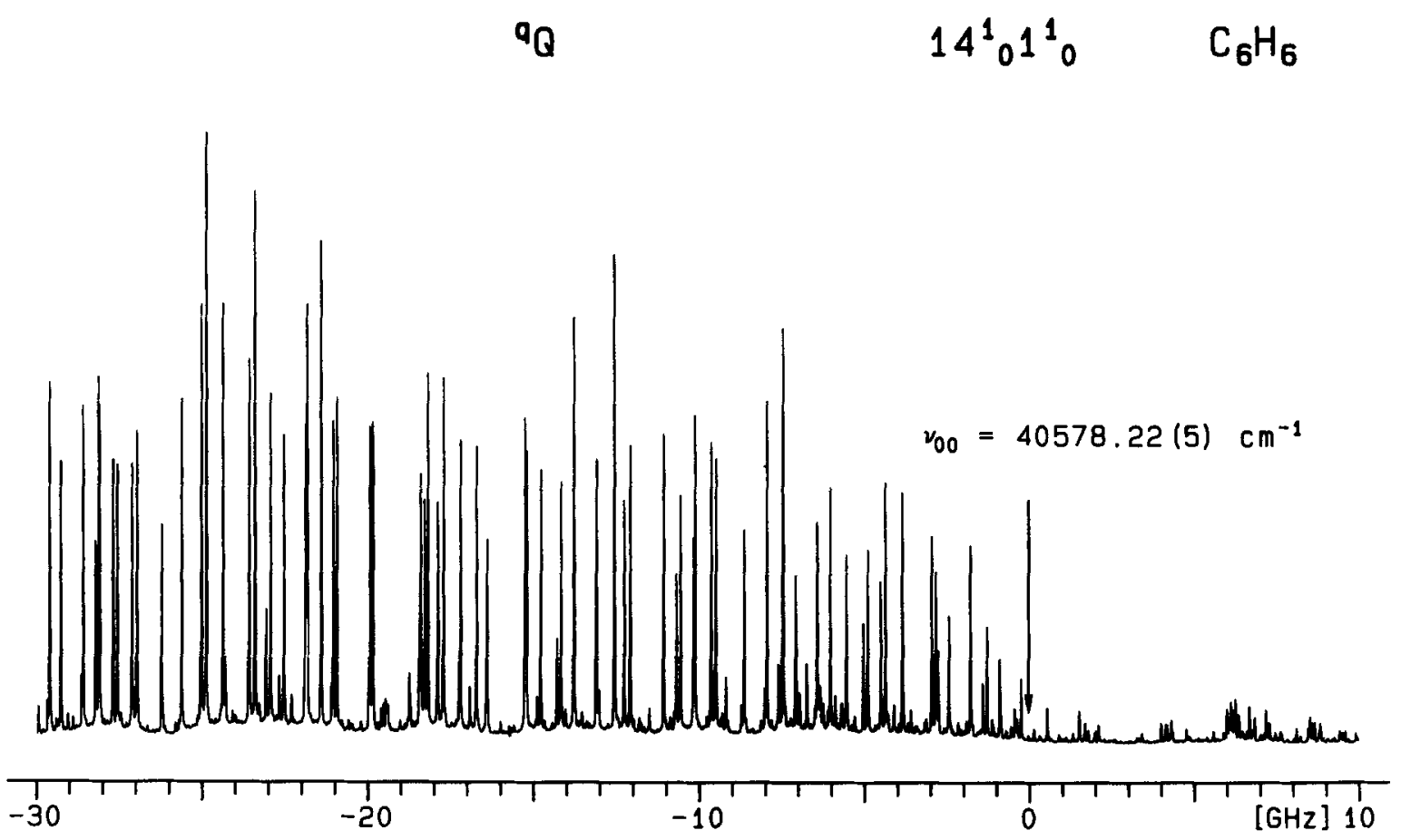

FIG. 7. Part of the Doppler-free two-photon spectrum of the $Q$ branch of the $14_{0}^{1} 1_{0}^{1}$ band in $\mathrm{C}_{6} \mathrm{H}_{6}$. The rotationless origin $v_{00}$ is marked by an arrow. 
gy of $2492 \mathrm{~cm}^{-1}$. The total density of background states is 18 $1 / \mathrm{cm}^{-1}$. The rotational line structure of this band has been investigated in great detail and only few perturbed lines were found in the blue part of the $Q$ branch. ${ }^{5}$ However, this band has a "shadow" band shifted $28.8 \mathrm{~cm}^{-1}$ to the blue. It was assigned as the $14_{0}^{1} 16_{0}^{1} 17_{0}^{1}$ band and gains its intensity from a long-range Fermi resonance with the $14^{1} 1^{1}$ state. ${ }^{26}$ This long-range Fermi resonance does not affect the relative positions of the rotational lines within the band contour, but there is an overlap of both bands similar to that described above. Part of the spectrum is shown in Fig. 7. To the blue of the rotationless origin the weaker rotational line structure of the $14_{0}^{1} 16_{0}^{1} 17_{0}^{1}$ band is seen which continues into the $14_{0}^{1} 1_{0}^{1}$ band and causes a stack of additional low intensity lines. Due to this additional band the total number of lines in the selected frequency range ( 0 to $-120 \mathrm{GHz}$ ) is about twice (531) that of the $14_{0}^{1}$ fundamental band (277) (see Table II).

The line intensity distribution for the range 0 to -120 $\mathrm{GHz}$ is shown in the upper part of Fig. 8. There is again a symmetric peak with maximum at the intensity 1 a.u. which was found to be typical for an unperturbed band [see Figs. 4(b), 4(d) ]. This is in excellent agreement with the weakly perturbed character of this band found from a detailed spectroscopic analysis. ${ }^{5}$ At very low intensities a strong increase of line numbers occurs. The origin of this peak is readily explained by the overlapping red wing of the $14_{0}^{1} 16_{0}^{1} 17_{0}^{1}$ band. In the lower part of Fig. 8 the intensity distribution of lines located to the blue of the rotationless origin is shown. There, only lines from the $14_{0}^{1} 16_{0}^{1} 17_{0}^{1}$ band are present. The position of the maximum agrees very well with the low intensity maximum in the upper part of Fig. 8.

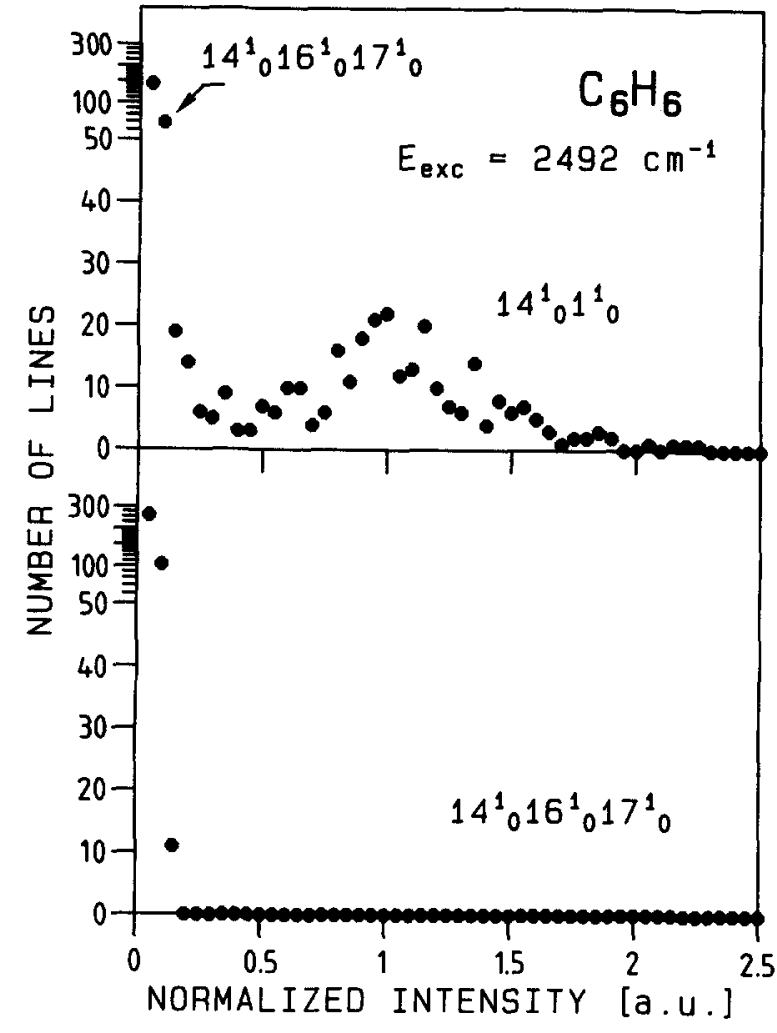

FIG. 8. Intensity distribution of lines between 0 and $-120 \mathrm{GHz}$ : Upper trace: In the $Q$ branch of the $14_{0}^{1} 1_{0}^{1}$ band in $\mathrm{C}_{6} \mathrm{H}_{6}$. The two peaks result from the lines of the two overlapping bands which are indicated in the figure. Lower trace: Of lines located to the blue of the rotationless origin of the $14_{0}^{1} 11_{0}^{1}$ band. Only lines of the $14_{0}^{1} 16_{0}^{1} 17_{0}^{1}$ band contribute. Note the change in scale for the vertical axis. For explanation of the intensity scale (horizontal axis) see the text.
${ }^{q_{Q}}$
$14^{1}$
$\mathrm{C}_{6} \mathrm{D}_{6}$
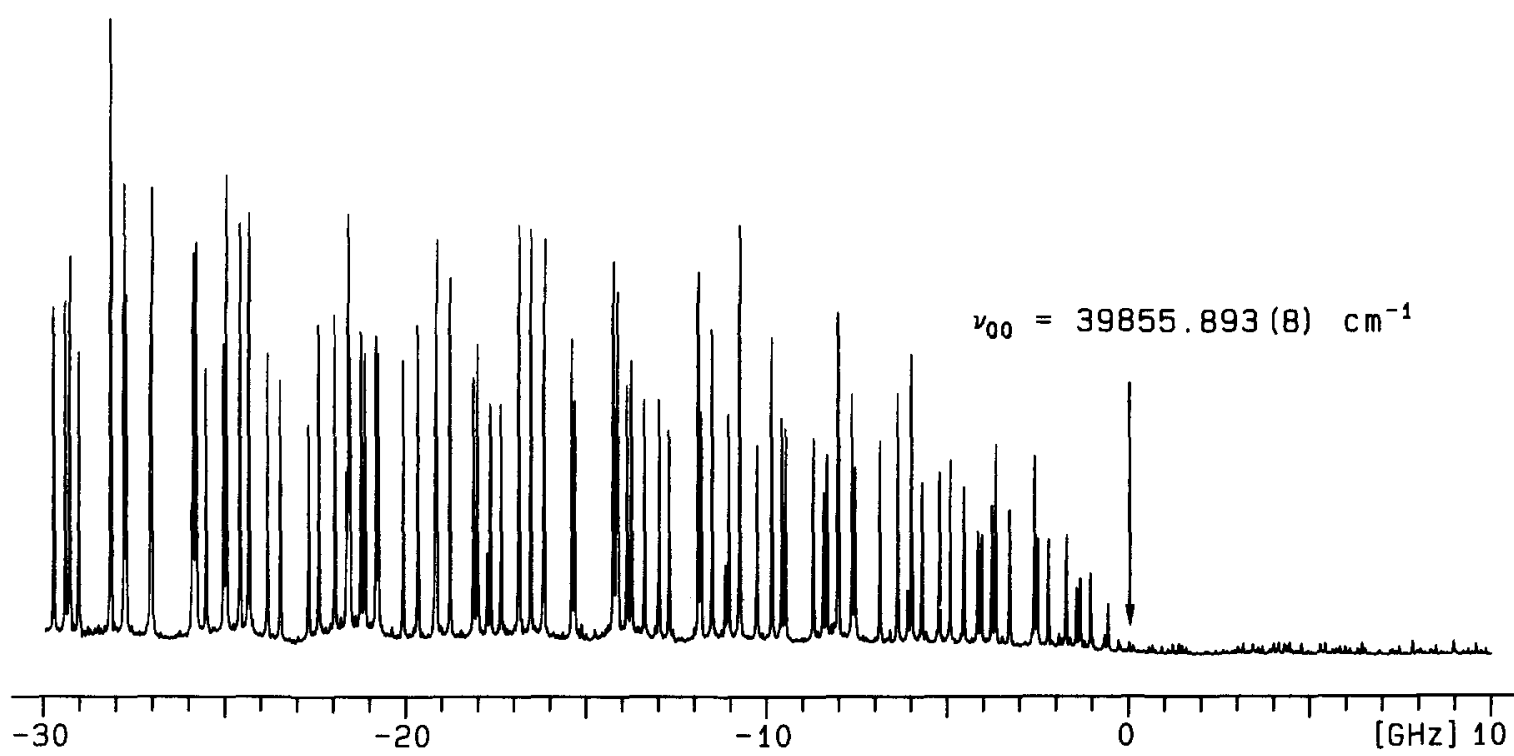

FIG. 9. Part of the Doppler-free two-photon spectrum of the $Q$ branch of the $14_{0}^{1}$ band in $C_{6} D_{6}$. The rotationless origin $v_{00}$ is marked by an arrow. 


\section{4. $14_{0}^{7}$ band In $C_{8} D_{0}$}

The line spectrum of the blue edge of the $Q$ branch of the $14_{0}^{1}$ band in $C_{6} D_{6}$ is shown in Fig. 9. The rotationless origin $v_{00}$ is located at $39855.893(8) \mathrm{cm}^{-1}$ two-photon energy and the final $14^{1}$ state is at an excess energy of $1567 \mathrm{~cm}^{-1}$. There the density of states is $41 / \mathrm{cm}^{-1}$. In the investigated spectral range ( 0 to $-120 \mathrm{GHz}$ ) nearly no perturbations were found and every line was assigned. ${ }^{35}$ The analysis leads to the rotational constants shown in Table I. No remarkable interfering contributions from another neighbored band are present, as can be seen by inspection of the range to the blue of the rotationless origin.

In Fig. 10 the corresponding line intensity distribution is shown and the data are given in Table II. The line intensity distribution is peaked near 1 a.u. and displays a symmetric shape. This is in line with the nonperturbed character of this band found from the detailed spectroscopic analysis.

\section{Summary for weakly perturbed bands}

In this section we have shown that the normalized line intensity distribution has a particular shape for nonperturbed or weakly perturbed bands. For the selected part of the rotational contour (blue edge of the $Q$ branch) it displays a symmetric peak with a maximum whose position is the same for the various totally symmetric two-photon bands under consideration. The number of lines is rapidly decreasing for smaller intensities. Due to the high dynamic range in our experiment, the statistics are good enough to even separate the contributions from overlapping bands. For the weakly perturbed bands studied so far, the characteristic intensity distribution of unperturbed bands was found also for this complex situation.

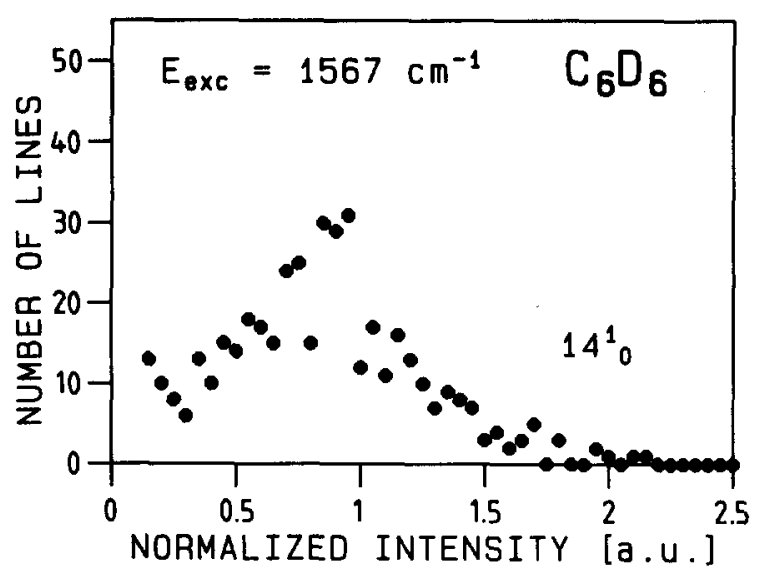

FIG. 10. Intensity distribution of lines between 0 and $-120 \mathrm{GHz}$ : in the $Q$ branch of the $144_{0}^{!}$band in $C_{6} D_{6}$. For explanation of the intensity scale see the text.

\section{Line intensity distribution of strongly perturbed bands}

In the preceding section bands at relatively small excess energy with low density of background states $(<181 /$ $\mathrm{cm}^{-1}$ ) have been investigated. Now we would like to study two bands at somewhat higher excess energy. Efforts have been made to analyze the rotational structure of these bands but an assignment of the rotational lines is, if at all, only possible for a few lines. Obviously, these bands are strongly perturbed and it is of interest to investigate whether the intensity distribution of these bands differs from that of the nonperturbed bands discussed in the preceding section.

Progression bands of two of the fundamental bands de-

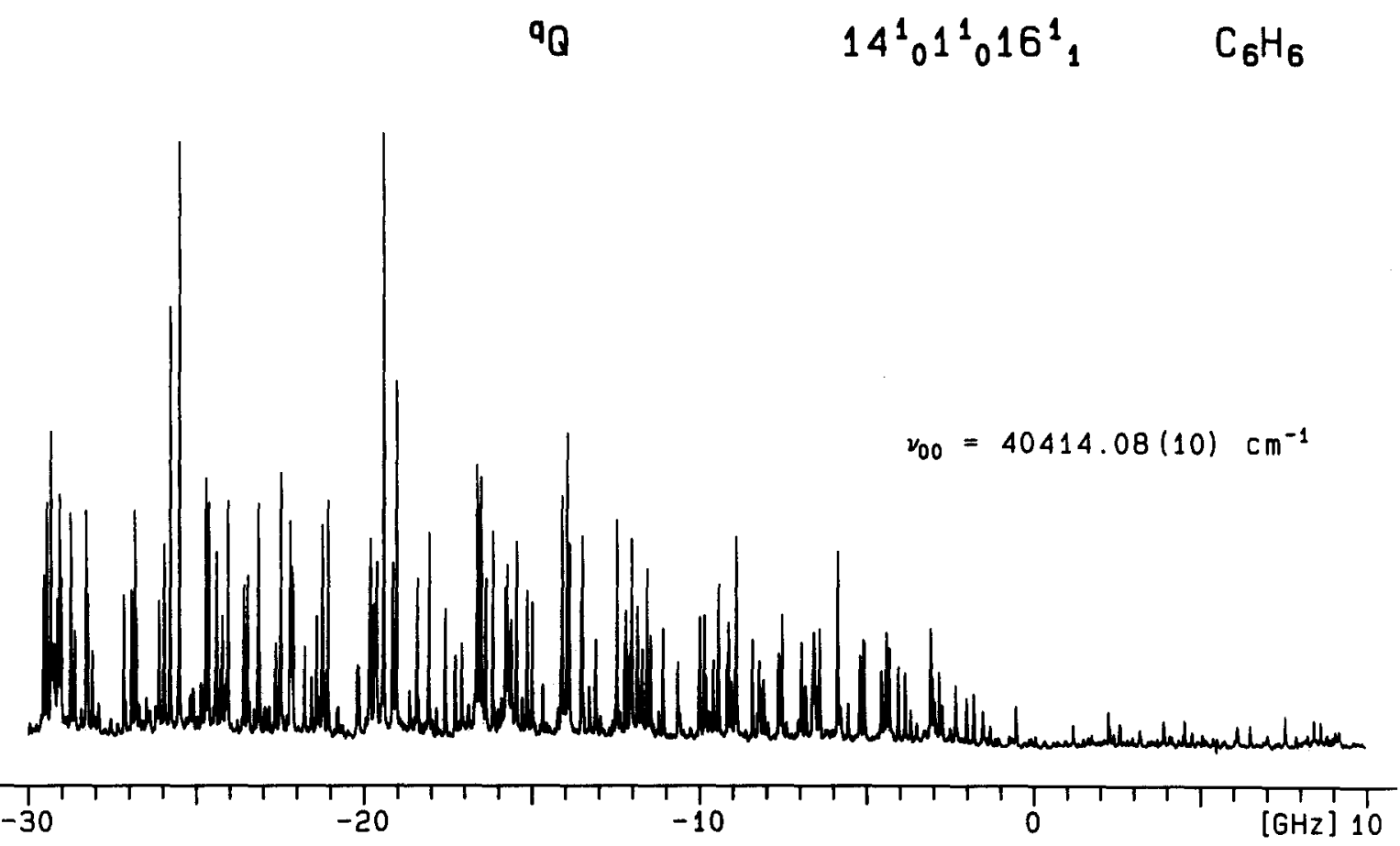

FIG. 11. Part of the Doppler-free two-photon spectrum of the $Q$ branch of the $14_{0}^{1} 1_{0}^{1} 16_{1}^{1}$ band in $C_{6} H_{6}$. 
scribed and analyzed above have been selected. In the progression band one quantum of the totally symmetric C-C stretch vibration $v_{1}\left(923 \mathrm{~cm}^{-1}\right)$ is excited in addition to the fundamental vibration. ${ }^{36}$ Since there is no change in the selection rules and only a small change in the rotational constants. the rotational structure is expected to be similar to that of the corresponding fundamental band. If no perturbations were present also the line intensity distribution should resemble that of the fundamental band.

\section{1. $14_{0}^{1} 116_{o}^{\prime}$ band in $\mathrm{C}_{6} \mathrm{H}_{6}$}

The rotationless origin $v_{00}$ of the $14_{0}^{1} 1_{0}^{1} 16_{1}^{1}$ band in $\mathrm{C}_{6} \mathrm{H}_{6}$ is at a two-photon excitation energy of $40414.08(10)$ $\mathrm{cm}^{-1}$ and the final $14^{1} 1^{1} 16^{1}$ state is at an excess energy of $2727 \mathrm{~cm}^{-1}$ with a total density of states of $321 / \mathrm{cm}^{-1}$. The measured blue edge of the $Q$ branch of this band is shown in Fig. 11. To the blue of the rotationless origin there are only tiny contributions from the neighbored $14_{0}^{1} 1_{0}^{1} 11_{1}^{1}$ band. This is in agreement with the low resolution results of Sur et al. ${ }^{28}$ who found that the ratio between the intensities of the $14_{0}^{1} 16_{1}^{1}$ and the $14_{0}^{1} 11_{1}^{1}$ bands is smaller than the corresponding ratio for the $14_{0}^{1} 1{ }_{0}^{1} 16_{1}^{1}$ and $14_{0}^{1} 1_{0}^{1} 11_{1}^{1}$ bands. The band displays a complicated rotational structure. Somers et al. ${ }^{34}$ could only analyze and assign part of the rotational lines and they concluded that in addition to the $l$-type doubling which is already present in the $14_{0}^{1} 16_{1}^{1}$ fundamental band this band is strongly perturbed.

In the upper part of Fig. 12 the line intensity distribution is shown. It results from the statistical analysis of 827 lines located in the selected frequency range of 0 to $-120 \mathrm{GHz}$. This is about the same number of lines as observed in the $14_{0}^{1} 16_{1}^{1}$ fundamental band. However, it has to be taken into account that about $30 \%$ of the lines in that case originate from the overlapping $14_{0}^{1} 11_{1}^{1}$ band. For the $14_{0}^{1} 1_{0}^{1} 16_{1}^{1}$ band no remarkable contributions from the $14_{0}^{1} 1_{0}^{1} 11_{1}^{1}$ band are present and the lines found in excess of twice 277 lines (see $14_{0}^{1}$ band) expected at most for the $l$-type doubling can only be ascribed to perturbations present in this band.

This conclusion drawn from the total number of lines is strongly supported by the intensity distribution. It completely differs from the distributions of the weakly perturbed bands shown in the preceding section. There is no longer a maximum but instead the number of lines increases monotonically with decreasing intensity. In the lower part of Fig. 12 the normalized line intensity distribution for the frequency range 0 to $+35.75 \mathrm{GHz}$ to the blue of the rotationless origin is shown. It reflects the small contribution from the overlapping very weak $14_{0}^{1} 1_{0}^{1} 11_{1}^{1}$ band (see above). It is seen that these contributions may only affect the very low intensity part ( $<0.2 \mathrm{a} . \mathrm{u}$.) of the intensity distribution in the range 0 to $-120 \mathrm{GHz}$. Hence, we conclude that the many small lines in the intensity range $0.2-1$ are the result of many perturbations occurring in this band. This is in qualitative agreement with the strongly perturbed character of this band found from the detailed spectral analysis. ${ }^{34}$

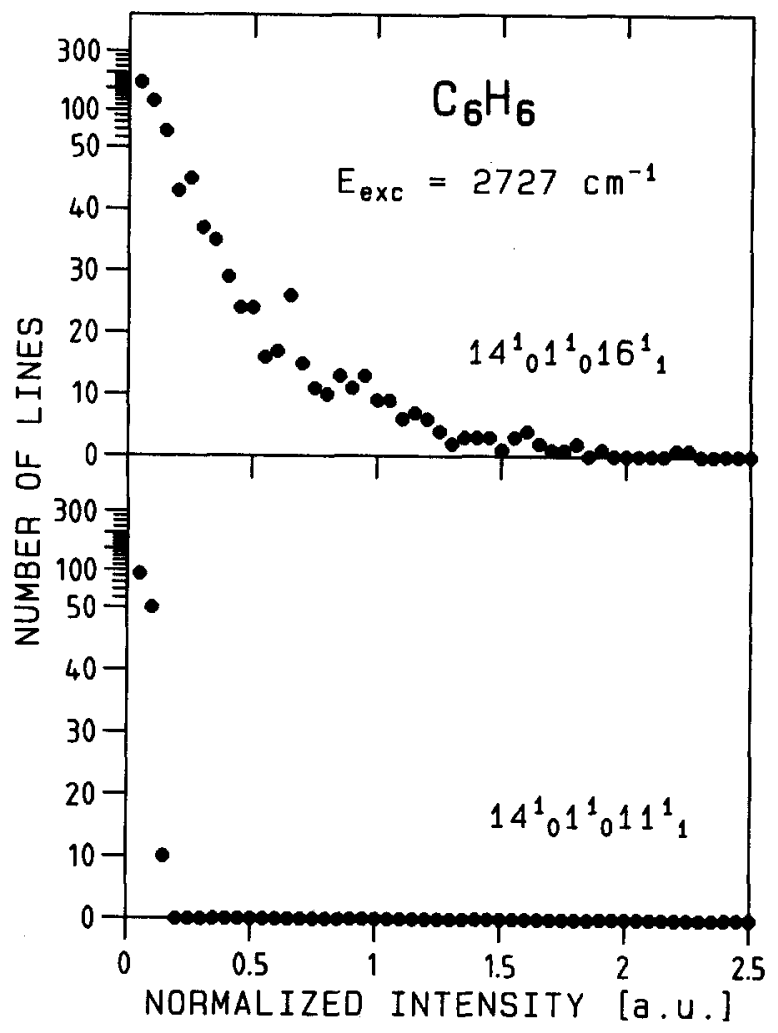

FIG. 12. Intensity distribution of lines between 0 and $-120 \mathrm{GHz}$ : Upper trace: in the $Q$ branch of the $14_{0}^{1} 1_{0}^{1} 16_{1}^{1}$ band in $\mathrm{C}_{6} \mathrm{H}_{6}$. Lower trace: of lines located to the blue of the rotationless origin of the $14_{0}^{1} 1_{0}^{1} 16_{1}^{1}$ band. Only lines of the $14_{0}^{1} 1_{0}^{1} 11_{1}^{1}$ band contribute. Note the change in the scale of the vertical axis. For explanation of the intensity scale (horizontal axis) see the text.

\section{2. $14_{0}^{1} 1_{0}^{1}$ band in $C_{6} D_{6}$}

The rotationless origin $v_{00}$ of the $14_{0}^{1} 1_{0}^{1}$ band in $C_{6} D_{6}$ is located at a two-photon energy of $40733.84(10) \mathrm{cm}^{-1}$ and the final $14^{1} 1^{1}$ state has an excess energy of $2445 \mathrm{~cm}^{-1}$. Even though the excess energy is smaller by $47 \mathrm{~cm}^{-1}$ than that of the corresponding $14^{1} 1^{1}$ state in $\mathrm{C}_{6} \mathrm{H}_{6}$ the total density of background states is $601 / \mathrm{cm}^{-1}$ and larger by a factor of 3 . The blue edge of this band is shown in Fig. 13. To the blue of the rotationless origin it is seen that there is no remarkable contribution from the $14_{0}^{1} 10_{0}^{2}$ band which is located 33.2 $\mathrm{cm}^{-1}$ to the blue of the $14_{0}^{1} 1_{0}^{1}$ band. We have not been able to analyze the rotational structure in Fig. 13 on the basis of a symmetric rotor approximation. Only the $J=K$ lines which are the strongest lines in the isotropic $Q$ branch seem to be in the neighborhood of the expected positions. We therefore concluded that this band is strongly perturbed due to couplings to the many background states.

The line intensity distribution is shown in the upper part of Fig. 14. It results from the intensity statistics of 740 lines which is a factor of 1.57 more than the total number of lines in the corresponding part of the fundamental band. The shape of the intensity distribution is quite different from the distribution of the $14_{0}^{1}$ fundamental band shown in Fig. 10. It displays no maximum at the intensity position ( 1 a.u.) expected for an unperturbed band. Instead, there is a monotonically increasing number of smaller lines. Only the very small 


$$
{ }^{\mathrm{a}} \mathrm{Q} \quad 14^{1}{ }_{0} 1_{0}^{1} \quad \mathrm{C}_{6} \mathrm{D}_{6}
$$

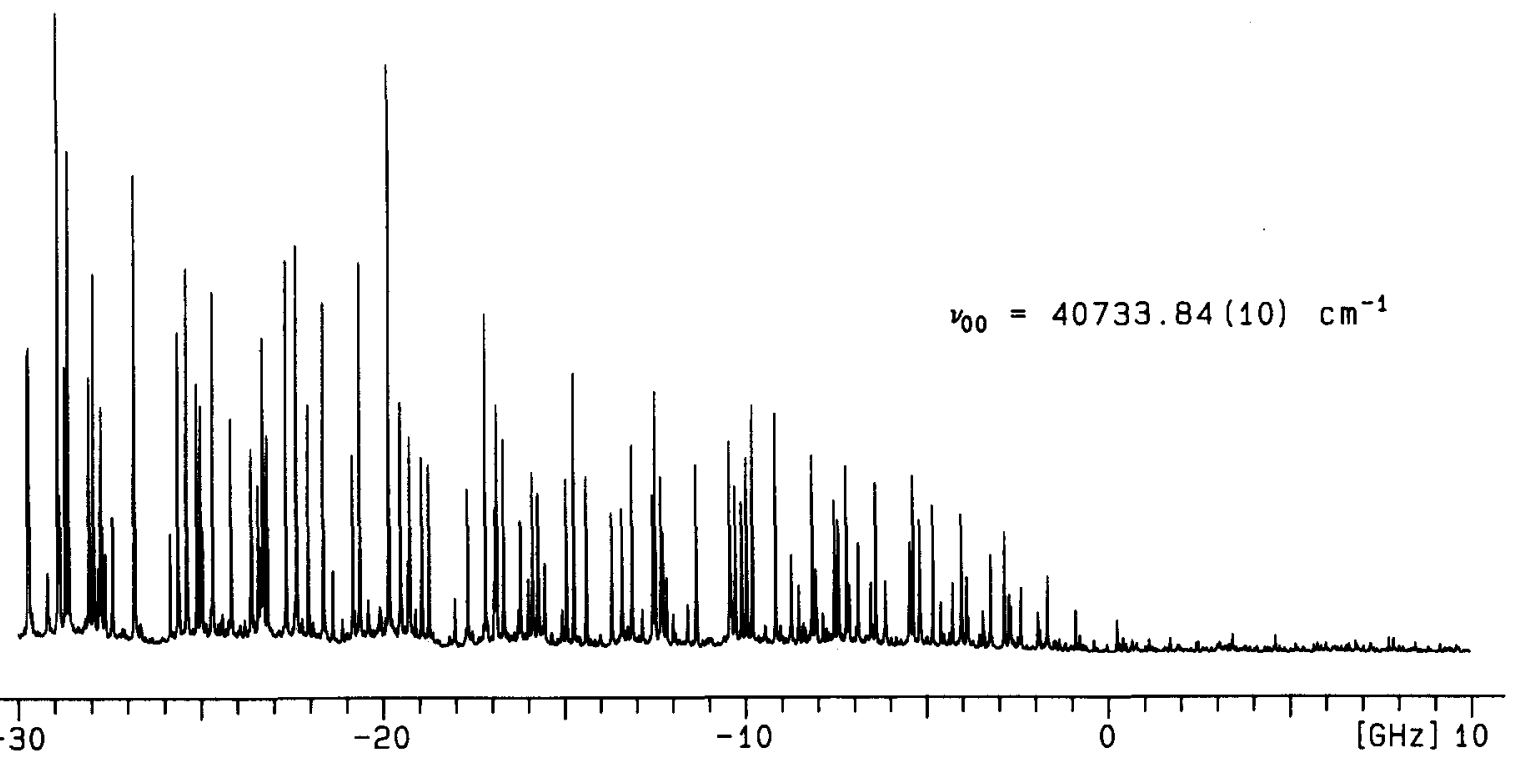

FIG. 13. Part of the Doppler-free two-photon spectrum of the $Q$ branch in the $14_{0}^{1} 1_{0}^{1}$ band in $C_{6} D_{6}$.

lines with an intensity smaller than 0.1 a.u. can be ascribed to the small contributions from the overlapping $14_{0}^{1} 10_{0}^{2}$ band. This can be seen from a comparison of the upper part with the lower part in Fig. 14 which displays the intensity distri-

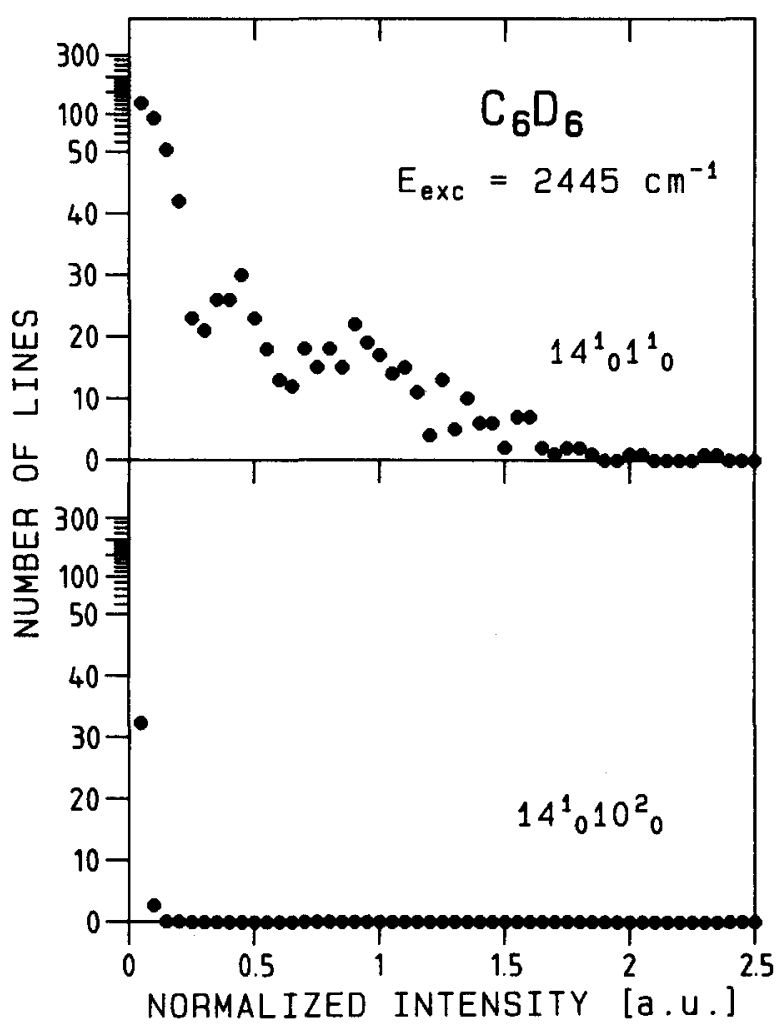

FIG. 14. Intensity distribution of lines between 0 and $-120 \mathrm{GHz}$ : Upper trace: In the $Q$ branch of the $14_{0}^{1} 1_{0}^{1}$ band in $\mathrm{C}_{6} \mathrm{D}_{6}$. Lower trace: Of lines located to the blue of the rotationless origin of the $14_{0}^{1} 1_{0}^{1}$ band. Only lines of the $14_{0}^{1} 10_{0}^{2}$ band contribute. Note the change in the scale of the vertical axis. For explanation of the intensity scale (horizontal axis) see the text. bution of lines in the frequency range 0 to $+44.7 \mathrm{GHz}$ to the blue of the rotationless origin, where only the $14_{0}^{1} 10_{0}^{2}$ band is present. The large number of low intensity lines and the increased total number of lines is expected for a strongly perturbed band.

In Table II, all experimental results and the theoretical results are summarized. It is interesting to see that the total counting rate varies for the different bands as they have been measured under different experimental conditions (laser power, etc.). The perfect theoretical simulation of the unperturbed bands is demonstrated by the good agreement of line numbers found in the frequency range under investigation for the experimental and the theoretical $14_{0}^{1}$ spectrum of $\mathrm{C}_{6} \mathrm{H}_{6}$. The two bands $14_{0}^{1} 1_{0}^{1} 16_{1}^{1}$ in $\mathrm{C}_{6} \mathrm{H}_{6}$ and $14_{0}^{1} 1_{0}^{1}$ in $\mathrm{C}_{6} \mathrm{D}_{6}$ with the highest density of states $\left(32\right.$ and $601 / \mathrm{cm}^{-1}$ ) display a principally different line intensity distribution " $p$ ". Unperturbed bands have a peaked intensity distribution with one maximum (" $m$ ") or two maxima (" $2 m$ ") if two unperturbed bands are overlapping.

\section{SUMMARY AND CONCLUSION}

Doppler-free two-photon spectroscopy provides completely resolved rotational line spectra of vibronic bands of large polyatomic molecules. This makes the same precision in spectroscopy feasible which hitherto has only been possible for much smaller systems. ${ }^{4}$ More important, a completely new situation arises which is typical for large systems with many vibrational degrees of freedom rather than for small molecules. In a large polyatomic system optically accessible light states with large Franck-Condon factors are embedded in a manifold of dark background states whose number is strongly increasing with excess energy. These background 
states are sometimes coupled to the light states by short range higher order Coriolis and/or anharmonic couplings. They produce perturbations in the rotational line spectrum of the light states. The degree of perturbance is expected to increase with the number of background states, i.e., with increasing vibrational excess energy.

In this work we presented several completely resolved line spectra of vibronic bands at different excess energies in the $S_{1}$ state of benzene. The bands are perturbed to a different degree and assignment of the rotational structure is not always possible. For this reason a statistical procedure has been applied to the line spectra and the intensity distribution of the lines has been investigated. It is found that there is a strong correlation between the degree of perturbation and the shape of the line intensity distribution. Intensity distributions of weakly perturbed bands differ strongly from that of perturbed bands. It is concluded that line intensity distributions are a powerful tool for statistical spectroscopy. In particular, on the basis of a line intensity distribution, a decision can be made whether a complicated assignment procedure is reasonable for a band under investigation.

Beyond that, it is interesting to discuss whether conclusions on the regular or irregular character of a spectrum can be drawn from the line intensity distributions presented in this work. The usual way to obtain information on this property is a statistics of line spacings. For an irregular spectrum a Wigner distribution and for a regular spectrum a Poisson distribution is expected. ${ }^{7,8}$ This type of statistical analysis cannot be applied to the spectra of this work since the spectrum contains many rotational sequence bands. This is due to the population of several thousands of ground state rotational levels at room temperature. The level spacing statistics would always lead to a Poisson distribution irrespective of the regularity or irregularity of the spectrum.

A different situation is present for the line intensity statistics. For an irregular spectrum, a Porter Thomas ${ }^{37}$ distribution is expected. In order to obtain information on the degree of irregularity, the line intensity distributions have to be compared with the theoretically predicted distribution. The theoretical procedure to investigate this question will be discussed in the subsequent part II of this work.

\section{ACKNOWLEDGMENTS}

The authors are indebted to Professor Schlag for his permanent interest. They would like to thank Professor Levine for stimulating discussions and U. Schubert for performing part of the measurements. Financial support from the Deutsche Forschungsgemeinschaft is greatfully acknowledged. H.S. would like to thank the Hanns-Seidel-Stiftung for graduate support.
${ }^{1}$ For a review, see C. S. Parmenter, Faraday Discuss. Chem. Soc. 75, 7 (1983).

${ }^{2}$ E. Riedle, H. J. Neusser, and E. W. Schlag, J. Chem. Phys. 75, 4231 (1981).

${ }^{3}$ E. Riedle and H. J. Neusser, J. Chem. Phys. 80, 4686 (1984).

${ }^{4} \mathrm{H}$. J. Neusser and E. Riedle, Comments At. Mol. Phys. 19, 331 (1987).

${ }^{5}$ E. Riedle, H. Stepp, and H. J. Neusser, Chem. Phys. Lett. 110, 452 (1984).

${ }^{6}$ U. Schubert, F. Giesemann, E. Riedle, and H. J. Neusser (in preparation). ${ }^{7}$ R. D. Levine, Adv. Chem. Phys. 70, 53 (1987).

${ }^{8}$ Th. Zimmermann, L. S. Cederbaum, H.-D. Meyer, and H. Köppel, J. Phys. Chem. 91, 4446 (1987).

${ }^{9}$ H. L. Dai, C. L. Korpa, J. L. Kinsey, and R. W. Field, J. Chem. Phys. 82, 1688 (1985).

${ }^{10} \mathrm{~J}$. P. Pique, Y. Chen, R. W. Field, and J. L. Kinsey, Phys. Rev. Lett. 58, 475 (1987).

${ }^{11}$ E. Abramson, R. W. Field, D. Imre, K. K. Innes, and J. L. Kinsey, J. Chem. Phys. 80, 2298 (1984); 83, 453 (1985); R. L. Sundberg, E. Abramson, J. L. Kinsey, and R. W. Field, ibid. 83, 466 (1985).

${ }^{12}$ G. Persch, E. Mehdizadeh, W. Demtröder, Th. Zimmermann, H. Köppel, and L. S. Cederbaum, Ber. Bunsenges. Phys. Chem. 92, 312 (1988); Th. Zimmermann, H. Köppel, L. S. Cederbaum, G. Persch, and W. Demtröder, Phys. Rev. Lett. 61, 3 (1988).

${ }^{13}$ S. L. Coy, K. K. Lehmann, and F. C. DeLucia, J. Chem. Phys. 85, 4297 (1986); K. K. Lehmann and S. L. Coy, ibid. 83, 3290 (1985).

${ }^{14}$ E. P. Peyroula, R. Jost, M. Lombardi, and P. Dupré, Chem. Phys. 102, 417 (1986).

${ }^{15}$ D. R. Guyer, W. F. Polik, and C. B. Moore, J. Chem. Phys. 84, 6519 (1986).

16J. Kommandeur, L. W. Meerts, Y. M. Engel, and R. D. Levine, J. Chem. Phys. 88, 6810 (1988).

${ }^{17}$ L. Leviandier, M. Lombardi, R. Jost, and J. P. Pique, Phys. Rev. Lett. 56, 2449 (1986); R. Jost and M. Lombardi, Lect. Notes Phys. 263, 72 (1986).

${ }^{18}$ J. H. Callomon, J. E. Parkin, and R. Lopez-Delgado, Chem. Phys. Lett. 13, 125 (1972).

${ }^{19}$ U. Schubert, E. Riedle, H. J. Neusser, and E. W. Schlag, J. Chem. Phys. 84, 6182 (1986).

${ }^{20}$ W. Dietz and S. F. Fischer, J. Chem. Phys. 87, 249 (1987).

${ }^{21}$ Y. M. Engel and R. D. Levine, J. Chem. Phys. 89, 4633 (1988).

${ }^{22}$ L. S. Vasilenko, V. P. Chebotayev, and A. V. Shishaev, JETP Lett. 12, 113 (1970).

${ }^{23}$ T. W. Hänsch and B. Couillaud, Opt. Commun. 35, 441 (1980).

${ }^{24}$ S. Gerstenkorn and P. Luc, Atlas du Spectre d'Absorption de la Molecule d'Iode (CNRS, Paris 1978).

${ }^{25}$ S. Gerstenkorn and P. Luc, Rev. Phys. Appl. 14, 791 (1979).

${ }^{26} \mathrm{~L}$. Wunsch, F. Metz, H. J. Neusser, and E. W. Schlag, J. Chem. Phys. 66, 386 (1977).

${ }^{27}$ F. Metz, W. E. Howard, L. Wunsch, H. J. Neusser, and E. W. Schlag, Proc. R. Soc. London Ser. A 363, 381 (1978).

${ }^{28}$ A. Sur, J. Knee, and P. Johnson, J. Chem. Phys. 77, 654 (1982).

${ }^{29}$ T. A. Stephenson, P. L. Radloff, and S. A. Rice, J. Chem. Phys 81, 1060 (1984).

${ }^{30}$ M. J. Robey and E. W. Schlag, J. Chem. Phys. 67, 2775 (1977).

${ }^{3}$ E. B. Wilson, Jr., J. C. Decius, and P. C. Cross, Molecular Vibrations (Dover, New York, 1980), p. 155.

${ }^{32}$ E. Riedle, H. Sieber, and H. J. Neusser (in preparation).

${ }^{33}$ U. Schubert, E. Riedle, and H. J. Neusser, J. Chem. Phys. 84, 5326 (1986).

${ }^{34}$ Doppler-free spectra of the $14_{0}^{1} 16_{1}^{1}$ and $14_{0}^{1} 11_{0}^{1} 16_{1}^{1}$ band were measured in our laboratory and analyzed by L. Somers and J. H. Callomon (manuscript in preparation).

${ }^{35}$ E. Riedle, Dissertation, TU München, 1984.

36J. H. Callomon, T. M. Dunn, and I. Mills, Philos. Trans. R. Soc. London Ser. A 259, 499 (1966).

${ }^{37}$ C. E. Porter and R. G. Thomas, Phys. Rev. 104, 483 (1956). 